
.

\title{
Unconventional Single-Molecule Conductance Behavior for a New Heterocyclic Anchoring Group: Pyrazolyl
}

\author{
I. Lucia Herrer, ${ }^{\dagger,}, \#$ Ali K. Ismael, ${ }^{\S, \|, \#}$ David C. Milán, ${ }^{\perp}$ Andrea Vezzoli, ${ }^{\perp}$ Santiago Martín, ${ }^{\dagger, \nabla}$

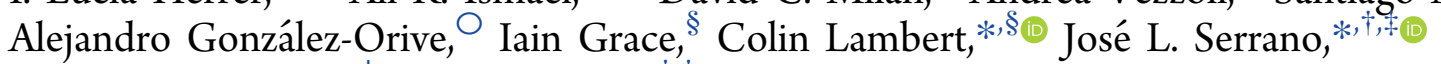 \\ Richard J. Nichols, ${ }^{*, \perp}$ and Pilar Cea, ${ }^{*,+}, \oplus$ \\ ${ }^{\dagger}$ Departamento de Química Física, Facultad de Ciencias, Universidad de Zaragoza, 50009 Zaragoza, Spain \\ ${ }^{\ddagger}$ Instituto de Nanociencia de Aragón (INA) and Laboratorio de Microscopias Avanzadas (LMA), edificio i+d Campus Río Ebro, \\ Universidad de Zaragoza, C/Mariano Esquillor, s/n, 50018 Zaragoza, Spain \\ ${ }^{\S}$ Department of Physics, University of Lancaster, Lancaster LA1 4YB, United Kingdom \\ "Department of Physics, College of Education for Pure Science, Tikrit University, Tikrit, Iraq \\ ${ }^{\perp}$ Department of Chemistry, University of Liverpool, Crown Street, Liverpool L69 7ZD, United Kingdom \\ ${ }^{\nabla}$ Instituto de Ciencias de Materiales de Aragón (ICMA), Universidad de Zaragoza-CSIC, 50009 Zaragoza, Spain

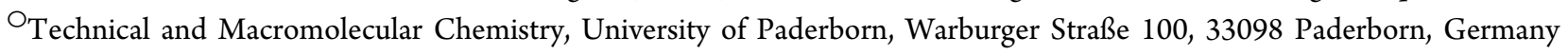 \\ Supporting Information
}

ABSTRACT: Electrical conductance across a molecular junction is strongly determined by the anchoring group of the molecule. Here we highlight the unusual behavior of 1,4-bis(1H-pyrazol-4-ylethynyl)benzene that exhibits unconventional junction current versus junction-stretching distance curves, which are peak-shaped and feature two conducting states of $2.3 \times 10^{-4} G_{0}$ and $3.4 \times 10^{-4} G_{0}$. A combination of theory and experiments is used to understand the conductance of single-molecule junctions featuring this new anchoring group, i.e., pyrazolyl. These results demonstrate that the pyrazolyl moiety changes its protonation state and contact binding during junction evolution and that it also binds in either end-on or facial geometries with gold contacts. The pyrazolyl moiety holds general interest as a contacting group, because this linkage leads to a strong double anchoring of the molecule to the gold electrode, resulting in enhanced conductance values.

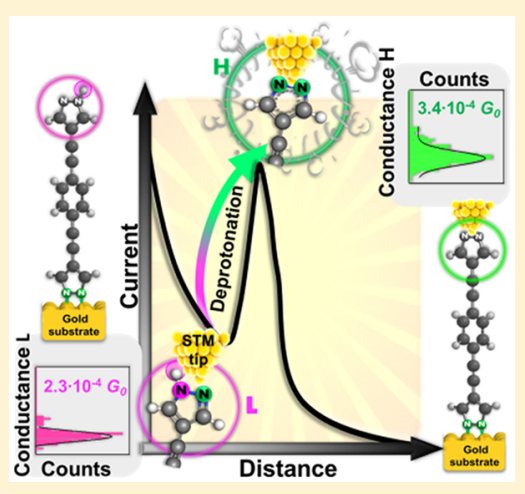

M olecular electronics has greatly advanced in the recent decades, demonstrating that molecular junctions can be extremely varied in their electrical responses and even mimic conventional circuit elements with wire-like, switching, diode, and transistor behavior. ${ }^{1,2}$ During the past 15 years, studies of single-molecule junctions have elucidated the fundamental mechanisms of charge transport through molecular bridges and led to new understanding of how the chemistry and electronic structure of the molecule and contacts can determine their electrical characteristics. The diversity of molecular bridges studied is now quite large and has ranged from organic to organometallic compounds and even metal atom chains (EMACs) with surrounding ligands and complex supramolecular assemblies. ${ }^{3}$ Because the coupling of the molecular bridges to the electrodes plays a central role, many studies have focused on the issue of anchor groups used to bind the molecular backbone to the enclosing electrical contacts. ${ }^{4,5}$ In this context, a large number of functional terminal groups have been explored, including thiols, ${ }^{6,7}$ selenols, ${ }^{8,9}$ dithiocarbamates, ${ }^{10,11}$ carbodithioates, ${ }^{12}$ amines, ${ }^{13,14}$ esters, ${ }^{15}$ cyanides, ${ }^{16,17}$ isocyanides, ${ }^{18}$ nitriles, ${ }^{19}$ carboxylic acids, ${ }^{15,20,21}$ dithiocarboxylic acids, $^{12}$ isothiocyanates, ${ }^{22}$ dimethylphos- phine, ${ }^{23}$ 4-(methylthio)phenyl groups, ${ }^{24}$ dihydrobenzo[b]thiophenes, ${ }^{25}$ thienyl rings, ${ }^{26,27}$ diphenylphosphine groups, ${ }^{28}$ trimethylsilylethynyl groups, ${ }^{29-31}$ viologens, ${ }^{32}$ tetrathiafulvalenes, ${ }^{33}$ or fullerenes. ${ }^{20,34,35}$ Some of these anchoring groups bind through a single terminal atom, while some feature multiple anchoring points. Here we aim to expand this family of multidentate anchor groups, because their multiple electrode bonding points might be advantageous for overall junction transmission and stability. ${ }^{36,37}$ Lower fluctuations due to stronger and more stable linkages to the substrate, ${ }^{38}$ as well as an increased thermal stability, ${ }^{10}$ may also be expected.

From the molecular electronics point of view, a strong and defined electronic coupling between the electrode, the anchor groups, and the rest of the molecule is required in order to promote a high transmission of the overall junction. ${ }^{35,38,37}$ However, in some cases, the steric requirements imposed by the design of molecules with two or more anchoring atoms or groups results in the disruption of $\pi$-conjugation in the

Received: July 1, 2018

Accepted: August 30, 2018

Published: August 30, 2018 
(a)
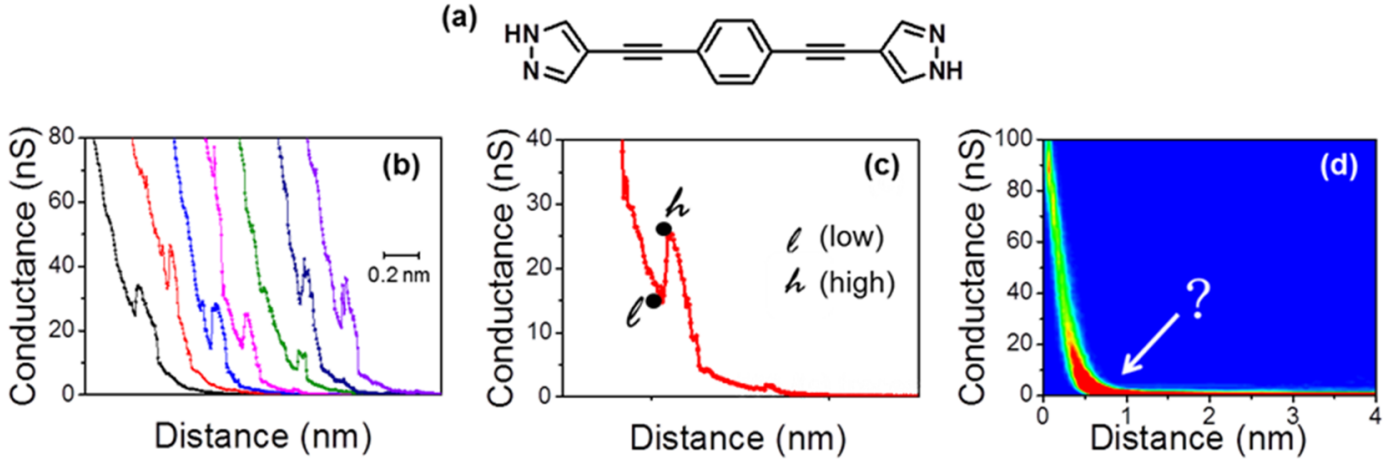

Figure 1. (a) Chemical structure of 1,4-bis(1H-pyrazol-4-ylethynyl)benzene, compound 1. (b) Seven representative $I(s)$ curves showing peak-like shape events. These curves were shifted horizontally for clarity. (c) A representative $I(s)$ trace obtained for compound 1 with high and low conductance points labeled. (d) 2D histogram obtained from $500 \mathrm{I}(\mathrm{s})$ curves recorded for $\mathbf{1}$ as shown in panel b.

a)
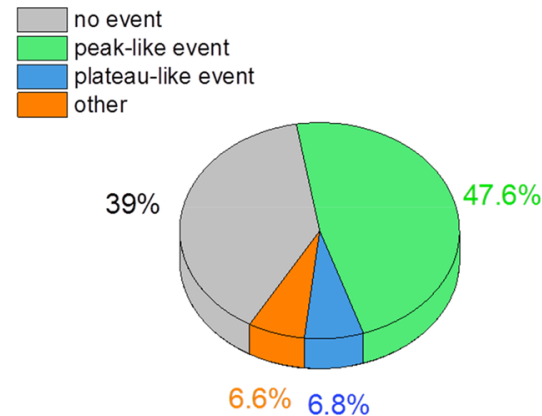

b)
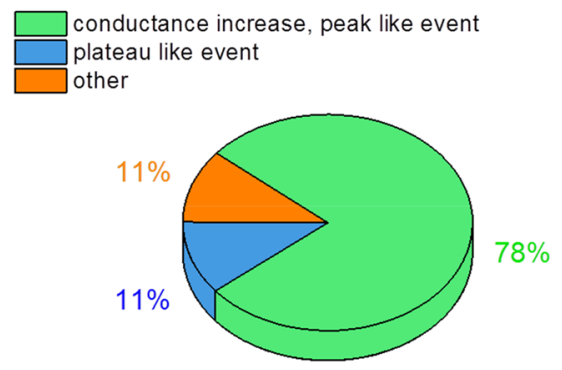

Figure 2. Junction formation statistics for 1000 unselected $I(s)$ recorded traces of compound 1. (a) Percentages corresponding to each of the observed indicated events. (b) Percentages determined considering only traces where molecular junctions are considered to have been formed. "Other" includes $I(s)$ curves exhibiting rather small plateaus (significantly lower than 0.1 nm) or noisy curves where the peak could not be clearly distinguished.

molecular structure, which compromises electrical transmission of the junction. However, interest in the topic is growing fast, and examples of multidentate platforms with potential uses in molecular electronics have already been published..$^{10,11,39-41}$ In this context, the exploration of multidentate materials has focused mainly on bidentate platforms such as dithiols, ${ }^{42-44}$ diols (cathecol), ${ }^{45}$ carbodithioates, ${ }^{12,46,47}$ dithiocarbamates, ${ }^{48-50}$ and norbornyldithiol. ${ }^{51,52}$ A few examples of tridentate $^{53-58}$ and tetradentate molecular platforms ${ }^{59}$ have also been reported.

In what follows, we explore new multidentate molecular junctions with the expectation of developing ligands that bind by a fundamentally different mode and which therefore could result in an increase in the molecular conductance. The focus of this present work is on the electrical behavior of compound 1 (1,4-bis(1H-pyrazol-4-ylethynyl)benzene, Figure 1a). Compound $\mathbf{1}$ is symmetrical along its longitudinal axis with two terminal pyrazolyl groups that, to the best of our knowledge, have not been used before as anchor groups in molecular wirebased materials. The electrical properties of compound 1 were studied using scanning tunneling microscopy (STM) combined with the $I(s)$ method and the break-junction (BJ) methods $^{60,61}$ in mesitylene solution (see the Supporting Information for further details). In these measurements, a clean flame-annealed $\mathrm{Au}(111)$ substrate was the bottom electrode and the STM tip (gold) was the top contact electrode. Initially, $500 \mathrm{I}(\mathrm{s})$ curves were recorded using a setpoint current $\left(I_{0}\right)$ of $60 \mathrm{nA}$ and a bias voltage $\left(V_{\text {bias }}\right)$ of $-0.6 \mathrm{~V}$. It is well-known that $I(s)$ traces in single-molecule measurements typically show a plateau shape as represented in Figure
S1a. The analysis of plateau-like $I(s)$ curves is schematically shown in Figure $S 1 b$ and allows the construction of conductance histograms, whose maxima correspond to the most probable value for the single-molecule conductance. However, when the shape of the event is not plateau-like, ${ }^{62}$ such a method (sequentially shown in Figure S1) cannot be applied. This is the case for compound 1, where traces do not exhibit the typical plateau-like shape. As can be seen in Figure 1 , a discernible plateau featuring current-distance $(I(s))$ curves are generally not observed for this molecular bridge. Instead the $I(s)$ curves exhibit peak-like events before junction breaking, while the typical 2D histogram (Figure 1d) does not show any clear feature that provides a reliable value for the single-molecule conductance.

To perform a comprehensive statistical analysis, $1000 \mathrm{I}(\mathrm{s})$ curves were recorded, including traces with all shape events of molecular junction formation and also traces without any event (neither peaks nor plateaus). In order to statistically represent this complex data set, the results obtained from these 1000 traces, where each curve was individually analyzed, are summarized in the pie diagrams shown in Figure 2. According to this statistical analysis, molecule-probe junctions occur ca. $60 \%$ of the time when the tip is retracted, and it was found that almost $80 \%$ of the conductance traces exhibited peak-like events; therefore, construction of common data histograms was ruled out. Instead, a bespoke analysis has been made to classify the low and high current values of $I(s)$ traces featuring peaks. As illustrated in Figures 1c,d, to construct this "lowhigh" (1-h) analysis of the individual traces, two different points were marked at the peak-like event: $l$, which corresponds to the 


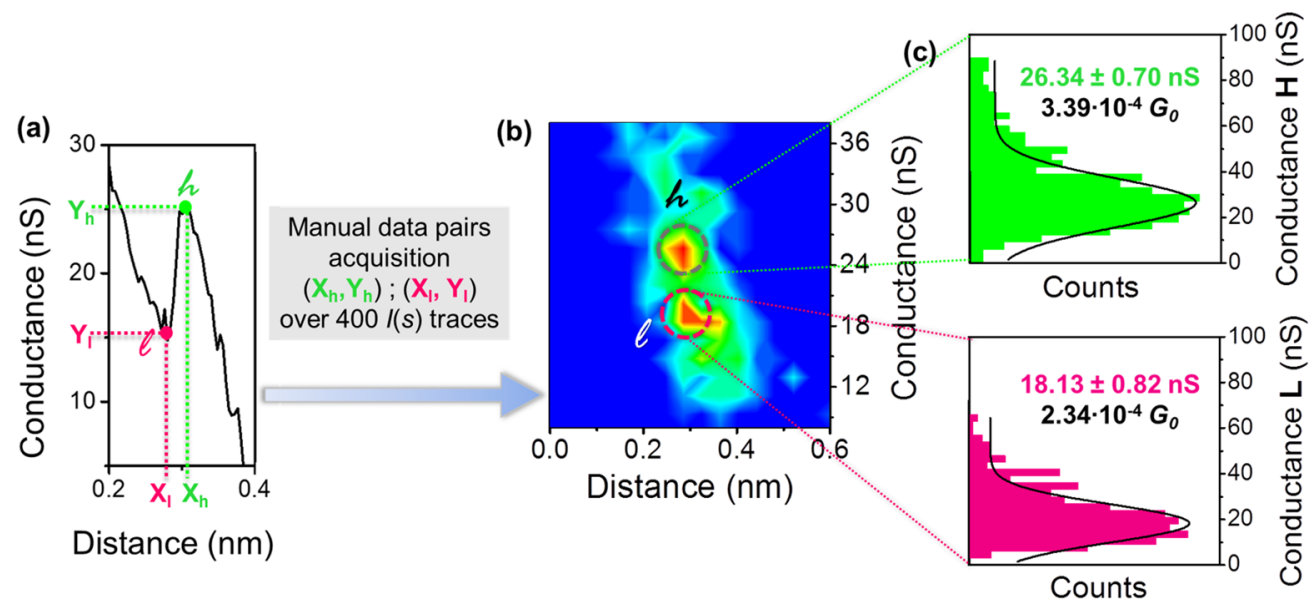

Figure 3. (a) Scheme illustrating the data analysis of more than $400 I(s)$ recorded traces showing a peak-like event for compound 1. (b) 2D histogram obtained from these $I(s)$ traces displaying the $\boldsymbol{l}$ and $\boldsymbol{h}$ states. (c) $1 \mathrm{D}$ histograms obtained for $\boldsymbol{l}$ and $\boldsymbol{h}$ conductance states of compound $\mathbf{l}$ and their conductance values.

low conductance through the molecular bridge, and $h$, representing the position where the maximum conductance value is reached.

As shown in Figure $1 \mathrm{c}$ there is a sudden conductance increase between the points $l$ and $\boldsymbol{h}$. Because these two points are spatially separated by just a small distance, they represent two different conductance states arising from the same pyrazolyl anchor group. With the aim of describing both states, the following procedure was used to analyze the recorded data: for each $I(s)$ trace containing a peak-like event, the $(X, Y)$ coordinates of both states were determined; then, two-dimensional histograms were plotted from the complete data set of all these data pairs. This procedure, along with the resulting "2D $\boldsymbol{l}$ - $\boldsymbol{h}$ pair distribution histogram", is shown in Figure 3. The 2D $l-h$ pair distribution histogram (Figure $3 b$ ) shows two discernible conductance values. For the lowconductance state $l$, the value of the molecular conductance is $2.34 \times 10^{-4} G_{0}$, and for the high-conductance state $\boldsymbol{h}$, the value corresponds to $3.39 \times 10^{-4} G_{0}$, as indicated in Figure 3c. Both values represent a sizable increase in the conductance compared to single-molecule conductance (SMC) values described earlier for molecular wires with a comparable chemical structure and length but incorporating other terminal contacting groups (see Table SI for comparison purposes)..$^{21,63-71}$ In addition, these conductance values are comparable with those of molecular junctions based on the strong $\mathrm{C}-\mathrm{Au}$ linkage. ${ }^{72}$ In order to confirm that the conductance jumps are not simply a feature of the $I(s)$ method, the break junction (BJ) technique was also employed to study the electrical properties of $\mathbf{1}$. The peak-like events observed in the $I(s)$ curves were also detected using the $\mathrm{BJ}$ method (further details can be found in the Supporting Information, Figures S3 and S4).

An increase of the conductance of some molecular junctions when they are stretched has been reported before. ${ }^{73}$ To elucidate the origin of the peak-like events in the $I(s)$ traces of compound 1, transport calculations using the density functional code SIESTA ${ }^{74}$ combined with the quantum transport code Gollum $^{75}$ were performed. Initially, the optimum geometries of the isolated molecules were obtained by relaxing the molecules until all forces on the atoms were lower than $0.05 \mathrm{eV} \cdot \AA^{-1}$. A double- $\zeta$ plus polarization orbital basis set and norm-conserving pseudopotentials were used. An energy cutoff of 250 Ry defined the real space grid, and the local density approximation (LDA) was chosen as the exchange-correlation functional. The results were also computed using GGA, and it was found that the resulting transmission functions were comparable with those obtained using LDA. To simulate an electrode tip formed during a break-junction experiment, ${ }^{76-79}$ electrodes were constructed from 6 layers of $\mathrm{Au}(111)$, each containing 30 gold atoms, with one of the leads terminated by a pyramid of gold atoms. ${ }^{80,81}$ Previous studies of the evolution of conductance-distance curves during junction breaking ${ }^{82,83}$ suggest that initially a planar terminal group binds cofacially to a pyramidal electrode tip, as shown in Figure 4 (top right). Then as the electrode gap increases, the terminal group is pulled toward a binding configuration involving only a single $\mathrm{Au}$ atom at the tip of the electrode, before it is finally detached from the electrode. In what follows, to understand the effect of retaining or losing the proton attached to one of the nitrogens of the pyrazolyl moiety, the electrical conductance of junctions with cofacially bound protonated pyrazolyl moiety (denoted $\left.P_{\text {cof }}\right)$ and deprotonated pyrazolyl unit $\left(D_{\text {cof }}\right)$ were computed. The term "co-facially-bound" refers to the pyrazolyl group aligning itself face-on to the side of the gold pyramidal tip. In addition, the conductance of tip-bound protonated pyrazolyl moiety $\left(P_{\text {tip }}\right)$ and deprotonated pyrazolyl moiety $\left(D_{\text {tip }}\right)$ were also computed as shown in the top right scheme in Figure 4. After each molecular configuration between fixed gold electrodes was relaxed using an extended molecule approach (protonated and deprotonated, see Figure S5 and Video S1) in the tip-bound or direct configuration (Figure S6), the terminal $\mathrm{Au}-\mathrm{N}$ bond distance was found to be $2.5 \AA$ for the protonated molecule and $2.4 \AA$ for the deprotonated molecule (see Figure $\mathrm{S7}$ ). For the flat gold surface, the $\mathrm{Au}-\mathrm{N}-\mathrm{C}$ angle was close to $180^{\circ}$ in all cases. For the cofacial configuration, the terminal $\mathrm{Au}-\mathrm{N}$ bond distance was found to be $2.9 \AA$ for the protonated and $2.6 \AA$ for the deprotonated molecule (see Figure S8). The pyrazolyl anchor group binds favorably to gold leads (direct contact), with a binding energy of $2.5 \mathrm{eV}$ for the deprotonated molecule and $0.5 \mathrm{eV}$ for the protonated molecule, whereas binding energies for cofacial contacts are $0.6 \mathrm{eV}$ for the protonated molecule and $1.9 \mathrm{eV}$ for the deprotonated molecule. The transmission coefficients for each of these binding coefficients are presented in Figure 4. The energetic location of frontier orbitals relative to the Fermi energy of 


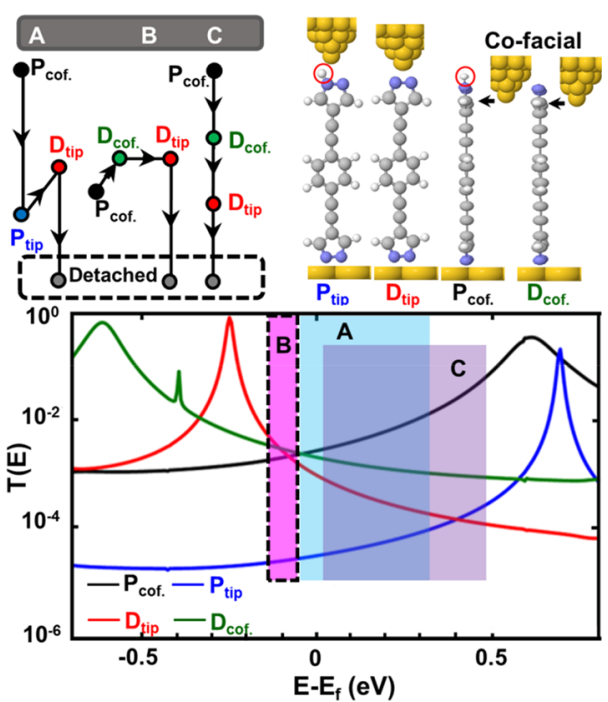

Figure 4. (Top right panel) Four binding configurations: a protonated molecule attached to a gold tip $\left(P_{\text {tip }}\right)$; a deprotonated molecule attached to a gold tip $\left(D_{\text {tip }}\right)$; a protonated molecule attached cofacially to a gold tip $\left(P_{\text {cof. }}\right)$, and a deprotonated molecule attached cofacially to a gold tip $\left(D_{\text {cof. }}\right)$. (Top left panel) A representation of the series of conductances measured for the three possible scenarios. Scenario A: The molecule passes through the sequence $P_{\text {cof. }} \rightarrow P_{\text {tip }} \rightarrow$ $D_{\text {tip }}$ and $E_{\mathrm{F}}$ lies within the blue region shown in the lower panel. Scenario B: The molecule passes through the sequence $P_{\text {cof. }} \rightarrow D_{\text {cof. }} \rightarrow$ $D_{\text {tip }}$ and $E_{\mathrm{F}}$ lies near the black-dashed vertical rectangle shown in the lower panel. Scenario $C$ : The molecule passes through the sequence $P_{\text {cof. }} \rightarrow D_{\text {cof. }} \rightarrow D_{\text {tip }}$ and $E_{\mathrm{F}}$ lies in the purple band shown in the lower panel, purple range. (Lower panel) Transmission coefficients $T(E)$ for the four binding configurations $P_{\text {tip }}, D_{\text {tip }}, P_{\text {cof., }}$, and $D_{\text {cof }}$.

electrodes is likely to vary from one pulling curve to the next pulling curve because of the stochastic nature of the junctionmaking and -breaking processes and therefore the energetic alignment of injected electrons relative to frontier orbitals will likewise exhibit broad variations. Hence, rather than consider a fixed single Fermi energy value (relative to the frontier levels), we consider how different broad energy ranges can lead to different behaviors in the transmission curves. The energy ranges are chosen on an ad hoc basis to illustrate the different experimental scenarios. The experimentally observed "high" and "low" conductance or no event are considered against the three possible scenarios (A, B, and C) shown in the top left panel of Figure 4 and detailed as follows:

Under scenario $\mathbf{A}$, the junction starts with a protonated cofacial binding configuration $\left(P_{\text {cof. }}\right)$ that evolves to a noncofacial protonated pyrazolyl group binding to the tip $\left(P_{\text {tip }}\right)$ and then evolves to a deprotonated binding to the tip $\left(D_{\text {tip }}\right)$, and eventually the molecule detaches. Provided that the energy of the injected electrons lie within the light blue band shown in Figure 4 , the transmission coefficient then switches from the black to the blue and then to the red curve, and this then results in the electrical conductance exhibiting a peak-like event. This scenario works for a wide range of energies roughly from -0.05 to $0.32 \mathrm{eV}$ as shown in lower panel of Figure 4 (light blue region), which means that the probability of peaklike events is high, in agreement with the experimental data shown in Figure 2.

Under scenario B, the junction starts in a similar way to A $\left(P_{\text {cof. }}\right)$, that then evolves to $\left(D_{\text {cof. }}\right)$ followed by $\left(D_{\text {tip }}\right)$ and eventually detaches. However, in the energy range of this scenario, denoted by the dashed rectangle in magenta on the lower panel of Figure 4 , the conductance of $\left(P_{\text {cof. }}\right)$ is less than that of $\left(D_{\text {cof. }}\right)$ and $\left(D_{\text {tip }}\right)$. This scenario results in only a small change of the conductance when moving from $D_{\text {cof. }}$ to $D_{\text {tip }}$ and therefore corresponds to the plateaus in a limited number of the $I(s)$ curves. The probability of this scenario is much lower than scenario A, because the energy range of scenario $B$ is limited to around $-0.1 \mathrm{eV}$.

Under scenario $C$, the junction has the same starting point of $\mathrm{A}$ and $\mathrm{B}$ and follows the sequence $P_{\text {cof. }} \rightarrow D_{\text {cof. }} \rightarrow D_{\text {tip. }}$. However, the energy range of electrons is chosen to correspond to the purple range denoted as C in Figure 4, and therefore, the conductance decreases in a series of steps, with no clear plateau and no peak. Such a behavior is in agreement with the green $I(s)$ curve of Figure $1 \mathrm{~b}$ in the energy range from approximately 0 to $0.5 \mathrm{eV}$.

Because deprotonating the molecule changes the charge state of the molecule, detailed spin-dependent density functional theory (DFT) spin calculations were also carried out. As shown in Figures S15-S18, the same qualitative behavior was found for the spin-up and the spin-down configurations, which indicates that no spin polarization occurs.

To experimentally probe the tendency of the pyrazolyl group to deprotonate in the presence of gold, additional experiments were performed. Figure 5 (left panel) shows the XPS spectrum of the powder of 1 and also the XPS spectra of LangmuirBlodgett (LB) monolayers of compound 1 (a monolayer instead of a single molecule is of course needed to obtain a reliable signal in the XPS experiments because of the detection limits of the technique). LB monolayers were prepared and transferred to gold slides from a subphase of pure water (Millipore Milli-Q $18.2 \mathrm{M} \Omega \cdot \mathrm{cm}, \mathrm{pH} 5.6$ ) and an aqueous subphase of $\mathrm{NaOH}$ ( $\mathrm{pH} 11.0$ ); more details can be found in Figure S19. The XPS spectrum for the powder of $\mathbf{1}$ in the $\mathrm{N} 1 \mathrm{~s}$ region reveals two peaks at $400.70 \mathrm{eV}$ (due to the pyrrole-like nitrogen) and $399.55 \mathrm{eV}$ (attributable to the pyridine-like nitrogen). ${ }^{84-86}$ The XPS spectrum of the LB monolayer prepared from a pure water subphase shows a broad band whose deconvolution reveals the presence of 3 peaks at 400.63 and $399.58 \mathrm{eV}$ (pyridine and pyrrole-like atoms) and at 401.30 $\mathrm{eV}$ (attributable to the pyrazolyl moiety chemisorbed on gold assuming that the molecule has lost the hydrogen associated with the pyrrole-like nitrogen upon chemisorption). Finally, LB films transferred from a basic subphase exhibit only two peaks centered at $401.20 \mathrm{eV}$ (deprotonated pyrazole chemisorbed onto gold) and at $399.50 \mathrm{eV}$ (deprotonated pyrazole on the free terminal group of the molecule due to the basic media, i.e. the one that is not attached to the gold substrate). These results are consistent with a clear tendency of compound 1 to chemisorb on the gold substrate through the two equivalent nitrogen atoms of the pyrazolyl heterocycle, accompanied by a deprotonation step, which also implies the formation of a bidentate junction with, at least, the bottom electrode. Figure 5 (left panel) also provides information about the areas of each peak, expressed as relative percentages. The peak areas corresponding to the powder of compound $\mathbf{1}$ are consistent with a $1: 1$ proportion of the two types of nitrogens in the molecule. The areas of the peaks recorded for the LB films of 1 cannot be interpreted quantitatively because the area of the peaks in the XPS spectrum is very sensitive to the location of the involved atom in the film, and in this case the two external nitrogen atoms result in higher integrated 
XPS spectra

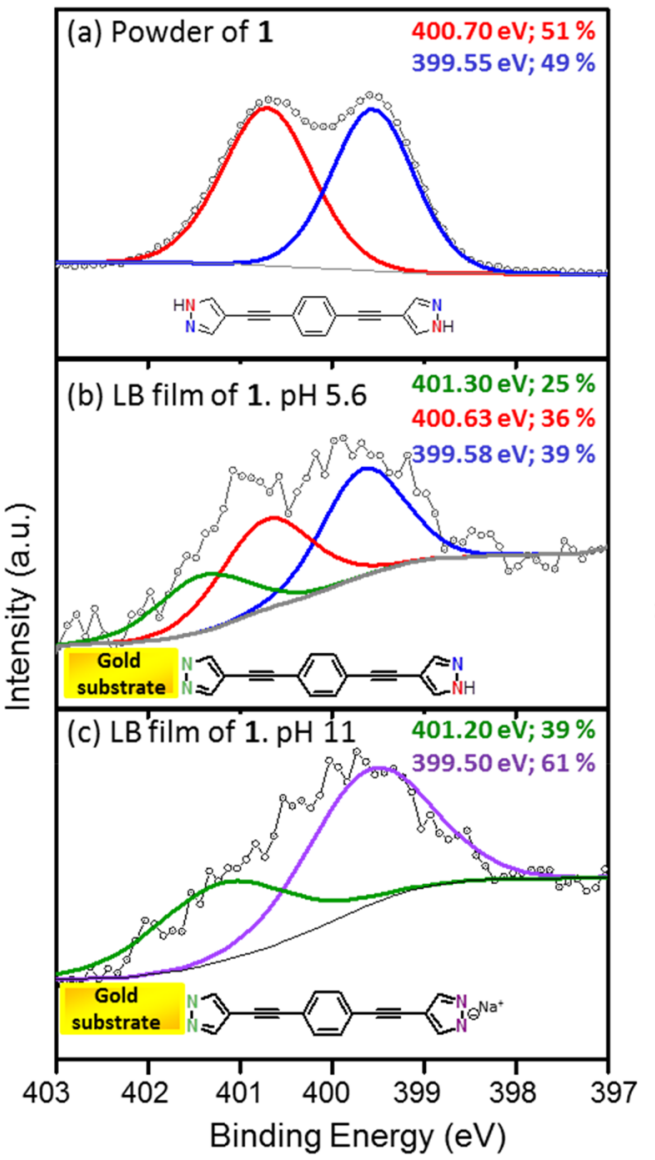

${ }^{1} \mathrm{H}-\mathrm{NMR}$ spectra

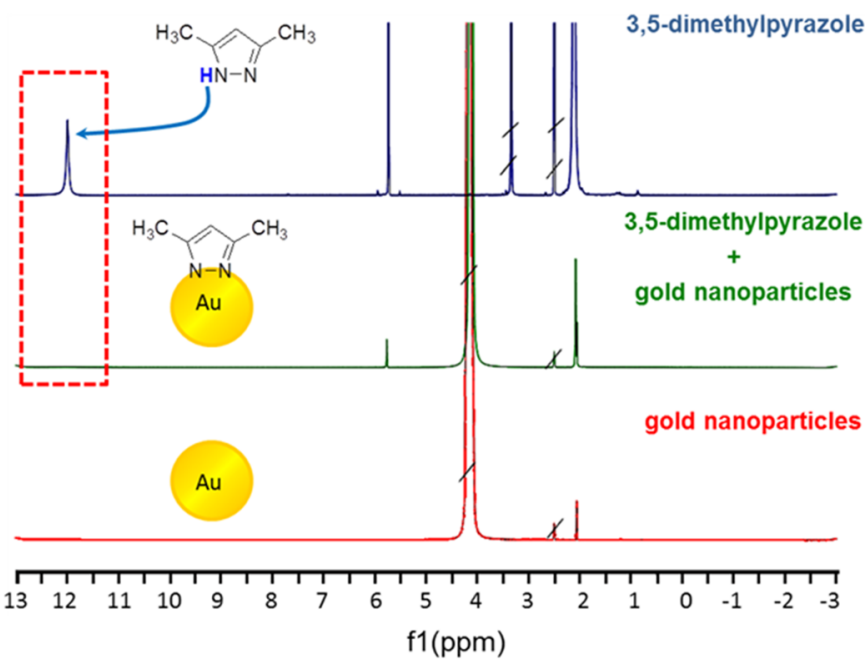

Figure 5. Experimental evidence of the deprotonation of the pyrazolyl group in the presence of gold. (Left panel) XPS spectra of the N 1s region for (a) the powder of $\mathbf{1}$; (b) a LB film of $\mathbf{1}$ on a gold substrate, which was transferred from a pure water subphase (pH 5.6); and (c) LB film of $\mathbf{1}$ on a gold substrate which was transferred from a basic subphase ( $\mathrm{pH} 11.0)$. (Right panel) ${ }^{1} \mathrm{H}$ NMR (400 MHz, DMSO- $d_{6}, \delta$ ppm) spectra of 3,5dimethylpyrazole (blue), uncapped gold nanoparticles (dispersed in Milli-Q water) (red), and a mixture of 3,5-dimethylpyrazole and uncapped gold nanoparticles (green). Crossed out lines correspond to solvents' signals.

intensities than the inner nitrogen atoms located and chemisorbed to the gold surface.

${ }^{1} \mathrm{H}$ NMR experiments were performed (Figure 5 right panel, with the spectra magnified in Figures S20-S22) for further confirmation of the chemisorption of the pyrazolyl heterocycle onto gold, given the capability of NMR spectroscopy as a method for structural analysis of the capping agents of nanoparticles. ${ }^{87}$ The compound 3,5-dimethylpyrazole was chosen for this experiment because it is the simplest molecule that contains the pyrazole ring and avoids the presence of other $\mathrm{H}$ atoms that could make the interpretation of the ${ }^{1} \mathrm{H}$ NMR spectra more difficult. The right panel in Figure 5 shows the ${ }^{1} \mathrm{H}$ NMR spectrum of 3,5-dimethylpyrazole in DMSO-d6 (deuterated dimethyl sulfoxide), which exhibits a signal at 12.0 ppm attributable to the pyrrole-like nitrogen proton of the pyrazole ring. The ${ }^{1} \mathrm{H}$ NMR spectrum of uncapped gold nanoparticles is also shown (Figure 5, right panel), and there are no signals at all in the $12 \mathrm{ppm}$ surroundings. Finally, the spectrum of a mixture of 3,5-dimethylpyrazole and gold nanoparticles (Figure 5, right panel) clearly shows the disappearance of the $12.0 \mathrm{ppm}$ signal, while the other ones from the 3,5-dimethylpyrazole remain in the ${ }^{1} \mathrm{H}$ NMR spectrum of the mixture. Therefore, this experiment further probes the tendency of the pyrazolyl moiety to be chemisorbed on gold through a chemical mechanism that involves a deprotonation process. From the XPS and also from the ${ }^{1} \mathrm{H}$ NMR experiments it can also be concluded that the deprotonated junction is stable and strong.

The role of hydrogen in interfaces of molecular junctions has been observed before. Thus, the electrical resistance of oligoanilines is known to be sensitive to $\mathrm{pH}$ in both bulk and molecular-scale devices because of reorganization and degradation of the junction after deprotonation, which results in a decay of the conductance by about 1 order of magnitude. ${ }^{88}$ Also, a conductance increase of seven times in carboxylateterminated monolayers versus carboxylic acid-terminated monolayers has been observed for the same molecular backbone. ${ }^{21}$ The change from carboxylic acid to carboxylate termination was also accompanied by a decrease in the effective barrier height of the tunneling junction. In that case, the deprotonation was chemically induced by a change in the $\mathrm{pH}$ of the media, and the increase of the conductance upon deprotonation was attributed to a stronger interaction between the tip of the STM and the $-\mathrm{COO}^{-}$terminal group in comparison with the $-\mathrm{COOH}$ termination, which additionally formed H-bonds between neighboring molecules. ${ }^{21}$ The sensitivity of dye molecules to environmental $\mathrm{pH}$ and the switching in conductance of the system under different $\mathrm{pH}$ values has been also reported. ${ }^{89}$ Recently, the conductance of vinylenedipyridine (44VDP) molecular junctions with $\mathrm{Ni}$ 
contacts was studied, found to be dependent on both the electrochemically applied gate voltage and the $\mathrm{pH}$ of the environment, and attributed to protonation-deprotonation of the pyridine anchor groups of 44VDP resulting in a change in the bonding at the metal-molecule interface. ${ }^{90}$

In summary, 1,4-bis(1H-pyrazol-4-ylethynyl)benzene represents a bidentate molecular platform that forms molecular junctions by chemisorbing on gold electrodes through a deprotonation process that results in a strong anchoring and high conductance values. This new anchor group exhibits highly unusual behavior, in which it changes its protonation state and contact binding during junction evolution, resulting in a sudden increase in conductance during the pulling process. These results open the door to the use of the pyrazolyl moiety as a binding group to produce effective electrical contacts between conjugated molecules and conducting substrates and also to exploit the switching behavior in the conductance values under protonation-deprotonation conditions for the construction of $\mathrm{pH}$ sensors based on molecular junctions.

\section{ASSOCIATED CONTENT}

\section{S Supporting Information}

The Supporting Information is available free of charge on the ACS Publications website at DOI: 10.1021/acs.jpclett.8b02051. Raw $I(s)$ and BJ data are available at the catalog: http://datacat.liverpool.ac.uk/460/.

Further discussion of the synthetic details, experimental procedures, and DFT calculations (PDF)

Full optimization process for the molecule together with the representative leads (Video S1) (AVI)

\section{AUTHOR INFORMATION}

\section{Corresponding Authors}

*E-mail: c.lambert@lancaster.ac.uk.

*E-mail: joseluis@unizar.es.

*E-mail: R.J.Nichols@liverpool.ac.uk.

*E-mail: pilarcea@unizar.es.

ORCID

Colin Lambert: 0000-0003-2332-9610

José L. Serrano: 0000-0001-9866-6633

Pilar Cea: 0000-0002-4729-9578

\section{Author Contributions}

${ }^{\#}$ L.I.H. and A.K.I. contributed equally to this work.

\section{Notes}

The authors declare no competing financial interest.

\section{ACKNOWLEDGMENTS}

P.C. and J.L.S. are grateful for financial assistance from Ministerio de Economia y Competitividad from Spain and fondos FEDER in the framework of projects MAT2016-78257$\mathrm{R}$ and CTQ2015-70174-P, respectively. J.L.S. also acknowledges the funded project Hierarchical Self Assembly of Polymeric Soft Systems, "SASSYPOL", from the seventh Framework Programme (CEE, ref-607602). L.I.H., S.M., J.L.S, and P.C. acknowledge support from DGA/Fondos FEDER (construyendo Europa desde Aragón) for funding PLATON (E31_17R) and CLIP (E47_17R) research groups. This work was additionally supported by the EPSRC "QuEEN" programme Grant EP/N017188/1. Further support from the UK EPSRC is acknowledged, through Grant Nos. EP/ M014452/1, EP/P027156/1, and EP/N03337X/1. Support from the European Commission is provided by the FET Open project 767187 - QuIET and the Iraqi Ministry of Higher Education (SL-20). A.K.I. acknowledges financial support from Tikrit University (Iraq). D.C.M., A.V. and R.J.N. gratefully acknowledge funding by EPSRC through grants EP/ M005046/1, EP/M014169/1, and EP/M029522/1.

\section{REFERENCES}

(1) Ratner, M. A brief history of molecular electronics. Nat. Nanotechnol. 2013, 8, 378-379.

(2) Aradhya, S. V.; Venkataraman, L. Single-molecule junctions beyond electronic transport. Nat. Nanotechnol. 2013, 8, 399-410.

(3) Nichols, R.; Higgins, S. Single-molecule electronics: chemical and analytical perspectives. Annu. Rev. Anal. Chem. 2015, 8, 389-417.

(4) Arroyo, C. R.; Leary, E.; Castellanos-Gómez, A.; RubioBollinger, G.; González, M. T.; Agrait, N. Influence of binding groups on molecular junction formation. J. Am. Chem. Soc. 2011, 133, 14313-14319.

(5) Jia, C.; Guo, X. Molecule-electrode interfaces in molecular electronic devices. Chem. Soc. Rev. 2013, 42, 5642-5660.

(6) Richter, L. J.; Yang, C. S.-C.; Wilson, P. T.; Hacker, C. A.; van Zee, R. D.; Stapleton, J. J.; Allara, D. L.; Yao, Y.; Tour, J. M. Optical characterization of oligo(phenylene-ethynylene) self-assembled monolayers on gold. J. Phys. Chem. B 2004, 108, 12547-12559.

(7) Häkkinen, H. The gold-sulfur interface at the nanoscale. Nat. Chem. 2012, 4, 443-455.

(8) Adaligil, E.; Shon, Y.-S.; Slowinski, K. Effect of Headgroup on Electrical Conductivity of Self-Assembled Monolayers on Mercury: nAlkanethiols versus n-Alkaneselenols. Langmuir 2010, 26, 15701573.

(9) Monnell, J. D.; Stapleton, J. J.; Dirk, S. M.; Reinerth, A.; Tour, J. M.; Allara, D. L.; Weiss, P. S. Relative conductances of alkaneselenolate and alkanethiolate monolayers on $\mathrm{Au}\{111\}$. J. Phys. Chem. B 2005, 109, 20343-20349.

(10) von Wrochem, F.; Gao, D.; Scholz, F.; Nothofer, H.-G.; Nelles, G.; Wessels, J. M. Efficient electronic coupling and improved stability with dithiocarbamate-based molecular junctions. Nat. Nanotechnol. 2010, 5, 618-624.

(11) Gao, D.; Scholz, F.; Nothofer, H. G.; Ford, W. E.; Scherf, U.; Wessels, J. M.; Yasuda, A.; von Wrochem, F. Fabrication of asymmetric molecular junctions by the oriented assembly of dithiocarbamate rectifiers. J. Am. Chem. Soc. 2011, 133, 5921-5930.

(12) Xing, Y.; Park, T. H.; Venkatramani, R.; Keinan, S.; Beratan, D. N.; Therien, M. J.; Borguet, E. Optimizing single-molecule conductivity of conjugated organic oligomers with carbodithioate linkers. J. Am. Chem. Soc. 2010, 132, 7946-7956.

(13) Lu, Q.; Yao, C.; Wang, X.; Wang, F. Enhancing molecular conductance of oligo(p-phenylene ethynylene)s by incorporating ferrocene into their backbones. J. Phys. Chem. C 2012, 116, 1785317861.

(14) Ashwell, G. J.; Williams, A.; Barnes, S. A.; Chappell, S. L.; Phillips, L. J.; Robinson, B. J.; Urasinska-Wojcik, B.; Wierzchowiec, P.; Gentle, I. R.; Wood, B. J. Self-assembly of amino-thiols via goldmitrogen links and consequence for in situ elongation of molecular wires on surface-modified electrodes. J. Phys. Chem. C 2011, 115, $4200-4208$.

(15) Villares, A.; Lydon, D. P.; Low, P. J.; Robinson, B. J.; Ashwell, G. J.; Royo, F. M.; Cea, P. Characterization and conductivity of Langmuir-Blodgett films prepared from an amine-substituted oligo(phenylene ethynylene). Chem. Mater. 2008, 20, 258-264.

(16) Mishchenko, A.; Zotti, L. A.; Vonlanthen, D.; Bürkle, M.; Pauly, F.; Cuevas, J. C.; Mayor, M.; Wandlowski, T. Single-molecule junctions based on nitrile-terminated biphenyls: a promising new anchoring group. J. Am. Chem. Soc. 2011, 133, 184-187.

(17) Zotti, L. A.; Kirchner, T.; Cuevas, J. C.; Pauly, F.; Huhn, T.; Scheer, E.; Erbe, A. Revealing the role of anchoring groups in the electrical conduction through single-molecule junctions. Small 2010, 6, 1529-1535. 
(18) Kim, B.; Beebe, J. M.; Jun, Y.; Zhu, X. Y.; Frisbie, C. D. Correlation between HOMO alignment and contact resistance in molecular junctions: Aromatic thiols versus aromatic isocyanides. J. Am. Chem. Soc. 2006, 128, 4970-4971.

(19) Beebe, J. M.; Engelkes, V. B.; Miller, L. L.; Frisbie, C. D. Contact resistance in metal-molecule-metal junctions based on aliphatic SAMs: Effects of surface linker and metal work function. J. Am. Chem. Soc. 2002, 124, 11268-11269.

(20) Martin, S.; Haiss, W.; Higgins, S.; Cea, P.; Lopez, M. C.; Nichols, R. J. A comprehensive study of the single molecule conductance of alpha,omega-dicarboxylic acid-terminated alkanes. J. Phys. Chem. C 2008, 112, 3941-3948.

(21) Ballesteros, L. M.; Martín, S.; Cortés, J.; Marqués-González, S.; Higgins, S. J.; Nichols, R. J.; Low, P. J.; Cea, P. Controlling the structural and electrical properties of diacid oligo(phenylene ethynylene) Langmuir-Blodgett films. Chem. - Eur. J. 2013, 19, 5352-5363.

(22) Ko, C.; Huang, M.; Fu, M.; Chen, C. Superior contact for single-molecule conductance: electronic coupling of thiolate and isothiocyanate on Pt, Pd, and Au. J. Am. Chem. Soc. 2010, 132, 756764.

(23) Park, Y. S.; Whalley, A. C.; Kamenetska, M.; Steigerwald, M. L.; Hybertsen, M. S.; Nuckolls, C.; Venkataraman, L. Contact chemistry and single-molecule conductance: A comparison of phosphines, methyl sulfides, and amines. J. Am. Chem. Soc. 2007, 129, 1576815769.

(24) Klausen, R. S.; Widawsky, J. R.; Steigerwald, M. L.; Venkataraman, L.; Nuckolls, C. Conductive molecular silicon. J. Am. Chem. Soc. 2012, 134, 4541-4544.

(25) Moreno-Garcia, P.; Gulcur, M.; Manrique, D. Z.; Pope, T.; Hong, W.; Kaliginedi, V.; Huang, C.; Batsanov, A. S.; Bryce, M. R.; Lambert, C. J.; et al. Single-molecule conductance of functionalized oligoynes: length dependence and junction evolution. J. Am. Chem. Soc. 2013, 135, 12228-12240.

(26) Arroyo, C. R.; Tarkuc, S.; Frisenda, R.; Seldenthuis, J. S.; Woerde, C. H. M.; Eelkema, R.; Grozema, F. C.; van der Zant, H. S. J. Signatures of quantum interference effects on charge transport through a single benzene ring. Angew. Chem., Int. Ed. 2013, 52, $3152-3155$.

(27) Bock, S.; Al-Owaedi, O. A.; Eaves, S. G.; Milan, D. C.; Lemmer, M.; Skelton, B. W.; Osorio, H. M.; Nichols, R. J.; Higgins, S.; Cea, P.; et al. Single-Molecule conductance studies of organometallic complexes bearing 3-Thienyl contacting groups. Chem. - Eur. J. 2017, 23, 2133.

(28) Parameswaran, R.; Widawsky, J. R.; Vázquez, H.; Park, Y. S.; Boardman, B. M.; Nuckolls, C.; Steigerwald, M. L.; Hybertsen, M. S.; Venkataraman, L. Reliable formation of single molecule junctions with air-stable diphenylphosphine linkers. J. Phys. Chem. Lett. 2010, 1, 2114-2119.

(29) Katsonis, N.; Marchenko, A.; Taillemite, S.; Fichou, D.; Chouraqui, G.; Aubert, C.; Malacria, M. A molecular approach to selfassembly of trimethylsilylacetylene derivatives on gold. Chem. - Eur. J. 2003, 9, 2574-2581.

(30) Katsonis, N.; Marchenko, A.; Fichou, D.; Barrett, N. Investigation on the nature of the chemical link between acetylenic organosilane self-assembled monolayers and $\mathrm{Au}(111)$ by means of synchrotron radiation photoelectron spectroscopy and scanning tunneling microscopy. Surf. Sci. 2008, 602, 9-16.

(31) Watcharinyanon, S.; Nilsson, D.; Moons, E.; Shaporenko, A.; Zharnikov, M.; Albinsson, B.; Mårtensson, J.; Johansson, L. S. O. A spectroscopic study of self-assembled monolayer of porphyrinfunctionalized oligo(phenyleneethynylene)s on gold: the influence of the anchor moiety. Phys. Chem. Chem. Phys. 2008, 10, 5264-5275. (32) Osorio, H. M.; Martin, S.; Milan, D. C.; Gonzalez-Orive, A.; Gluyas, J. B. G.; Higgins, S. J.; Low, P. J.; Nichols, R. J.; Cea, P. Influence of surface coverage on the formation of 4,4'- bipyridinium (viologen) single molecular junctions. J. Mater. Chem. C 2017, 5, 11717-11723.
(33) Jayamurugan, G.; Gowri, V.; Hernández, D.; Martín, S.; González-Orive, A.; Dengiz, C.; Dumele, O.; Pérez-Murano, F.; Gisselbrecht, J.-P.; Boudon, C.; et al. Design and synthesis of Aviram-Ratner-type dyads and rectification studies in LangmuirBlodgett (LB) films. Chem. - Eur. J. 2016, 22, 10539-10547.

(34) Atienza, C.; Martin, N.; Wielopolski, M.; Haworth, N.; Clark, T.; Guldi, D. M. Tuning electron transfer through p-penyleneethynylene molecular wires. Chem. Commun. 2006, No. 30, 3202-3204.

(35) Metzger, R. M. Unimolecular electronics and rectifiers. Synth. Met. 2009, 159, 2277-2281.

(36) Cea, P.; Ballesteros, L. M.; Martin, S. Nanofabrication techniques of highly organized monolayers sandwiched between two electrodes for molecular electronics. Nanofabrication 2014, 1, 96117.

(37) Valasek, M.; Lindner, M.; Mayor, M. Rigid multipodal platforms for metal surfaces. Beilstein J. Nanotechnol. 2016, 7, 374405.

(38) Chinwangso, P.; Jamison, A. C.; Lee, T. R. Multidentate adsorbates for self-assembled monolayer films. Acc. Chem. Res. 2011, 44, 511-519.

(39) Ditzler, L. R.; Karunatilaka, C.; Donuru, V. R.; Liu, H. Y.; Tivanski, A. V. Electromechanical properties of self-assembled monolayers of tetrathiafulvalene derivatives studied by conducting probe atomic force Microscopy. J. Phys. Chem. C 2010, 114, 44294435.

(40) James, D. K.; Tour, J. M. Molecular wires. Top. Curr. Chem. 2005, 257, 33-62.

(41) Terada, K.-i.; Nakamura, H.; Kanaizuka, K.; Haga, M. a.; Asai, Y.; Ishida, T. Long-range electron transport of ruthenium-centered multilayer films via a stepping-stone mechanism. ACS Nano 2012, 6 , 1988-1999.

(42) Rittikulsittichai, S.; Park, C. S.; Jamison, A. C.; Rodriguez, D.; Zenasni, O.; Lee, T. R. Bidentate aromatic thiols on gold: new insight regarding the influence of branching on the structure, packing, wetting, and stability of self-assembled monolayers on gold surfaces. Langmuir 2017, 33, 4396-4406.

(43) Sander, F.; Hermes, J. P.; Mayor, M.; Hamoudi, H.; Zharnikov, M. Add a third hook: S-acetyl protected oligophenylene pyridine dithiols as advanced precursors for self-assembled monolayers. Phys. Chem. Chem. Phys. 2013, 15, 2836-2846.

(44) Casalini, S.; Berto, M.; Leonardi, F.; Operamolla, A.; Bortolotti, C. A.; Borsari, M.; Sun, W.; Felice, R. D.; Corni, S.; Albonetti, C.; et al. Self-Assembly of mono-and bidentate oligoarylene thiols onto polycrystalline Au. Langmuir 2013, 29, 13198-13208.

(45) Ye, Q.; Wang, H.; Yu, B.; Zhou, F. Self-assembly of catecholic ferrocene and electrochemical behavior of its monolayer. RSC Adv. 2015, 5, 60090-60095.

(46) Tivanski, A. V.; He, Y.; Borguet, E.; Liu, H.; Walker, G. C.; Waldeck, D. H. Conjugated thiol linker for enhanced electrical conduction of gold-molecule contacts. J. Phys. Chem. B 2005, 109, $5398-5402$

(47) Li, Z. H.; Smeu, M.; Park, T. H.; Rawson, J.; Xing, Y. J.; Therien, M. J.; Ratner, M. A.; Borguet, E. Hapticity-dependent charge transport through carbodithioate-terminated 5,15-bis(phenylethynyl)porphinato zinc(II) complexes in metal-molecule-metal junctions. Nano Lett. 2014, 14, 5493-5499.

(48) von Wrochem, F.; Gao, D. Q.; Scholz, F.; Nothofer, H. G.; Nelles, G.; Wessels, J. M. Efficient electronic coupling and improved stability with dithiocarbamate-based molecular junctions. Nat. Nanotechnol. 2010, 5, 618-624.

(49) Gao, D.; Scholz, F.; Nothofer, H. G.; Ford, W. E.; Scherf, U.; Wessels, J. M.; Yasuda, A.; von Wrochem, F. Fabrication of asymmetric molecular junctions by the oriented assembly of dithiocarbamate rectifiers. J. Am. Chem. Soc. 2011, 133, 5921-5930.

(50) Sebechlebská, T.; Sebera, J.; Kolivoška, V.; Lindner, M.; Gasior, J.; Mészáros, G.; Valášek, M.; Mayor, M.; Hromadová, M. Investigation of the geometrical arrangement and single molecule charge transport in self-assembled monolayers of molecular towers 
based on tetraphenylmethane tripod. Electrochim. Acta 2017, 258, $1191-1200$.

(51) Darwish, N.; Diez-Perez, I.; Da Silva, P.; Tao, N. J.; Gooding, J. J.; Paddon-Row, M. N. Observation of electrochemically controlled quantum interference in a single anthraquinone-based norbornylogous bridge molecule. Angew. Chem., Int. Ed. 2012, 51, 3203-3206.

(52) Darwish, N.; Diez-Perez, I.; Guo, S. Y.; Tao, N. J.; Gooding, J. J.; Paddon-Row, M. N. Single molecular switches: electrochemical gating of a single anthraquinone-based norbornylogous bridge molecule. J. Phys. Chem. C 2012, 116, 21093-21097.

(53) Wei, Z.; Wang, X.; Borges, A.; Santella, M.; Li, T.; Sørensen, J. K.; Vanin, M.; Hu, W.; Liu, Y.; Ulstrup, J.; et al. Triazatriangulene as binding group for molecular electronics. Langmuir 2014, 30, 1486814876.

(54) Kuhn, S.; Jung, U.; Ulrich, S.; Herges, R.; Magnussen, O. Adlayers based on molecular platforms of trioxatriangulenium. Chem. Commun. 2011, 47, 8880-8882.

(55) Katano, S.; Kim, T. W.; Kitagawa, T.; Kawai, M. Tailoring electronic states of a single molecule using adamantane-based molecular tripods. Phys. Chem. Chem. Phys. 2013, 15, 14229-14233.

(56) Gerhard, L.; Edelmann, K.; Homberg, J.; Valasek, M.; Bahoosh, S. G.; Lukas, M.; Pauly, F.; Mayor, M.; Wulfhekel, W. An electrically actuated molecular toggle switch. Nat. Commun. 2017, 8, 14672.

(57) Ie, Y.; Hirose, T.; Nakamura, H.; Kiguchi, M.; Takagi, N.; Kawai, M.; Aso, Y. Nature of electron transport by pyridine-based tripodal anchors: potential for robust and conductive single-molecule junctions with gold electrodes. J. Am. Chem. Soc. 2011, 133, 30143022.

(58) Sakamoto, R.; Ohirabaru, Y.; Matsuoka, R.; Maeda, H.; Katagiri, S.; Nishihara, H. Orthogonal bis(terpyridine)-Fe(ii) metal complex oligomer wires on a tripodal scaffold: rapid electron transport. Chem. Commun. 2013, 49, 7108-7110.

(59) Kiguchi, M.; Takahashi, Y.; Fujii, S.; Takase, M.; Narita, T.; Iyoda, M.; Horikawa, M.; Naitoh, Y.; Nakamura, H. Additive electron pathway and nonadditive molecular conductance by using a multipodal bridging compound. J. Phys. Chem. C 2014, 118, 52755283.

(60) Xu, B. Q.; Tao, N. J. Measurement of single-molecule resistance by repeated formation of molecular junctions. Science 2003, 301, $1221-1223$

(61) Nichols, R. J.; Haiss, W.; Higgins, S. J.; Leary, E.; Martin, S.; Bethell, D. The experimental determination of the conductance of single molecules. Phys. Chem. Chem. Phys. 2010, 12, 2801-2815.

(62) Lemmer, M.; Inkpen, M. S.; Kornysheva, K.; Long, N. J.; Albrecht, T. Unsupervised vector-based classification of singlemolecule charge transport data. Nat. Commun. 2016, 7, 12922.

(63) Osorio, H. M.; Martin, S.; López, M. C.; Marques-Gonzalez, S.; Higgins, S. J.; Nichols, R. J.; Low, P. J.; Cea, P. Electrical characterization of single molecule and Langmuir-Blodgett monomolecular films of a pyridine-terminated oligo(phenylene-ethynylene) derivative. Beilstein J. Nanotechnol. 2015, 6, 1145-1157.

(64) Villares, A.; Pera, G.; Martín, S.; Nichols, R. J.; Lydon, D. P.; Applegarth, L.; Beeby, A.; Low, P. J.; Cea, P. Fabrication, characterization, and electrical properties of Langmuir-Blodgett films of an acid terminated phenylene-ethynylene oligomer. Chem. Mater. 2010, 22, 2041-2049.

(65) Ferradás, R. R.; Marqués-González, S.; Osorio, H. M.; Ferrer, J.; Cea, P.; Milan, D. C.; Vezzoli, A.; Higgins, S. J.; Nichols, R. J.; Low, P. J.; et al. Low variability of single-molecule conductance assisted by bulky metal-molecule contacts. RSC Adv. 2016, 6, 75111-75121.

(66) Lu, Q.; Liu, K.; Zhang, H.; Du, Z.; Wang, X.; Wang, F. From tunneling to hopping: a comprehensive investigation of charge transport mechanism in molecular junctions based on oligo(pphenylene ethynylene)s. ACS Nano 2009, 3, 3861-3868.

(67) Martin, S.; Grace, I.; Bryce, M. R.; Wang, C. S.; Jitchati, R.; Batsanov, A. S.; Higgins, S.; Lambert, C. J.; Nichols, R. J. Identifying diversity in nanoscale electrical break junctions. J. Am. Chem. Soc. 2010, 132, 9157-9164.
(68) Huber, R.; Gonzalez, M. T.; Wu, S.; Langer, M.; Grunder, S.; Horhoiu, V.; Mayor, M.; Bryce, M. R.; Wang, C. S.; Jitchati, R.; et al. Electrical conductance of conjugated oligorners at the single molecule level. J. Am. Chem. Soc. 2008, 130, 1080-1084.

(69) Tanaka, Y.; Kiguchi, M.; Akita, M. Inorganic and organometallic molecular wires for single-molecule devices. Chem. - Eur. J. 2017, 23, 4741-4749.

(70) Miguel, D.; Álvarez de Cienfuegos, L.; Martín-Lasanta, A.; Morcillo, S. P.; Zotti, L. A.; Leary, E.; Bürkle, M.; Asai, Y.; Jurado, R.; Cárdenas, D. J.; et al. Toward multiple conductance pathways with heterocycle-based oligo(phenyleneethynylene) derivatives. J. Am. Chem. Soc. 2015, 137, 13818-13826.

(71) Xiang, L.; Hines, T.; Palma, J. L.; Lu, X.; Mujica, V.; Ratner, M. A.; Zhou, G.; Tao, N. Non-exponential length dependence of conductance in iodide-terminated oligothiophene single-molecule tunneling junctions. J. Am. Chem. Soc. 2016, 138, 679-687.

(72) Olavarria-Contreras, I. J.; Perrin, M. L.; Chen, Z.; Klyatskaya, S.; Ruben, M.; van der Zant, H. S. J. C-Au covalently bonded molecular junctions using nonprotected alkynyl anchoring groups. J. Am. Chem. Soc. 2016, 138, 8465-8469.

(73) Saffarzadeh, A.; Demir, F.; Kirczenow, G. Mechanism of the enhanced conductance of a molecular junction under tensile stress. Phys. Rev. B: Condens. Matter Mater. Phys. 2014, 89, 045431.

(74) Soler, J. M.; Artacho, E.; Gale, J. D.; Garcia, A.; Junquera, J.; Ordejon, P.; Sanchez-Portal, D. The SIESTA method for ab initio order- N materials simulation. J. Phys.: Condens. Matter 2002, 14, 2745-2779.

(75) Ferrer, J.; Lambert, C. J.; García-Suárez, V. M.; Manrique, D. Z.; Visontai, D.; Oroszlany, L.; Rodríguez-Ferradás, R.; Grace, I.; Bailey, S. W. D.; Gillemot, K.; et al. GOLLUM: a next-generation simulation tool for electron, thermal and spin transport. New J. Phys. 2014, 16, 093029.

(76) Quek, S. Y.; Kamenetska, M.; Steigerwald, M. L.; Choi, H. J.; Louie, S. G.; Hybertsen, M. S.; Neaton, J. B.; Venkataraman, L. Mechanically controlled binary conductance switching of a singlemolecule junction. Nat. Nanotechnol. 2009, 4, 230-234.

(77) Kamenetska, M.; Quek, S. Y.; Whalley, A. C.; Steigerwald, M. L.; Choi, H. J.; Louie, S. G.; Nuckolls, C.; Hybertsen, M. S.; Neaton, J. B.; Venkataraman, L. Conductance and geometry of pyridine-linked single-molecule junctions. J. Am. Chem. Soc. 2010, 132, 6817-6821.

(78) Guo, S.; Hihath, J.; Díez-Pérez, I.; Tao, N. Measurement and statistical analysis of single-molecule current-voltage characteristics, transition voltage spectroscopy, and tunneling barrier Height. J. Am. Chem. Soc. 2011, 133, 19189-19197.

(79) Xu, B. Q.; Li, X. L.; Xiao, X. Y.; Sakaguchi, H.; Tao, N. J. Electromechanical and conductance switching properties of single oligothiophene molecules. Nano Lett. 2005, 5, 1491-1495.

(80) Dednam, W.; Sabater, C.; Fernandez, M. A.; Untiedt, C.; Palacios, J. J.; Caturla, M. J. Simulating cyclic loading of atomic-sized gold tips ongold surfaces via classical molecular dynamics and density functional theory transport calculations. Proc. 60th Annu. Conf. South African Inst. Phys., 2016; pp 487-492.

(81) Sabater, C.; Untiedt, C.; Palacios, J. J.; Caturla, M. J. Mechanical annealing of metallic electrodes at the atomic scale. Phys. Rev. Lett. 2012, 108, 205502.

(82) Davidson, R.; Al-Owaedi, O. A.; Milan, D. C.; Zeng, Q.; Tory, J.; Hartl, F.; Higgins, S. J.; Nichols, R. J.; Lambert, C. J.; Low, P. J. Effects of electrode-molecule binding and junction geometry on the single-molecule conductance of bis-2,2':6 $6^{\prime}, 2^{\prime \prime}$-Terpyridine-based complexes. Inorg. Chem. 2016, 55, 2691-2700.

(83) Mu, Y.; Zeng, Z.-Y.; Cheng, Y.; Chen, X.-R. Electronic transport properties of silicon carbide molecular junctions: firstprinciples study. RSC Adv. 2016, 6, 91453-91462.

(84) Katrib, A.; El-Rayyes, N. R.; Al-Kharafi, F. M. N 1s orbital binding energies of some pyrazole pyrazoline compounds by XPS. J. Electron Spectrosc. Relat. Phenom. 1983, 31, 317-321.

(85) Goswami, A.; Koskey, S.; Mukherjee, T.; Chyan, O. Study of pyrazole as copper corrosion inhibitor in alkaline post chemical 
mechanical polishing cleaning solution. ECS J. Solid State Sci. Technol. 2014, 3, P293-P297.

(86) Matsuoka, M.; Isotani, S.; Mansano, R. D.; Sucasaire, W.; Pinto, R. A. C.; Mittani, J. C. R.; Ogata, K.; Kuratani, N. X-Ray photoelectron spectroscopy and Raman spectroscopy studies on thin carbon nitride films deposited by reactive RF magnetron sputtering. World J. Nano Sci. Eng. 2012, 02, 92-102.

(87) Coelho, S. C.; Rangel, M.; Pereira, M. C.; Coelho, M. A. N.; Ivanova, G. Structural characterization of functionalized gold nanoparticles for drug delivery in cancer therapy: a NMR based approach. Phys. Chem. Chem. Phys. 2015, 17, 18971-18979.

(88) Feldman, A. K.; Steigerwald, M. L.; Guo, X.; Nuckolls, C. Molecular Electronic Devices Based on Single-Walled Carbon Nanotube Electrodes. Acc. Chem. Res. 2008, 41, 1731-1741.

(89) Li, Z.; Smeu, M.; Afsari, S.; Xing, Y.; Ratner, M. A.; Borguet, E. Single-Molecule Sensing of Environmental pH-an STM Break Junction and NEGF-DFT Approach. Angew. Chem. 2014, 126, 11161120.

(90) Brooke, R. J.; Szumski, D. S.; Vezzoli, A.; Higgins, S. J.; Nichols, R. J.; Schwarzacher, W. Dual Control of Molecular Conductance through $\mathrm{pH}$ and Potential in Single-Molecule Devices. Nano Lett. 2018, 18, 1317-1322. 